\title{
Serum zonulin is elevated in women with polycystic ovary syndrome and correlates with insulin resistance and severity of anovulation
}

\author{
Dongmei Zhang, Li Zhang, Fangzhi Yue, Yingying Zheng and Ryan Russell' \\ Department of Endocrinology, Xiangya Hospital, Central South University, 87 Xiangya Road, Changsha 410008, \\ Hunan, China and ${ }^{1}$ School of Nursing, University of California Los Angeles, Los Angeles, California, USA
}

Correspondence should be addressed to D Zhang

Email

drdmzhang@hotmail.com

\begin{abstract}
Objective: Evidence suggests that increased gut permeability may be associated with polycystic ovary syndrome (PCOS). Human zonulin is currently the only physiological mediator known to reversibly regulate gut permeability by disassembling intestinal tight junctions. So far, no data on serum zonulin levels in patients with PCOS are available. This study aimed to determine circulating serum zonulin levels in women with PCOS and discuss the relationship between zonulin, insulin resistance, and menstrual disorders in this group.

Design: A case-control study.

Methods: The study includes 78 women recently diagnosed with PCOS and 63 age-matched healthy controls recruited. Serum zonulin levels were determined by ELISA. Insulin resistance was assessed by homeostasis model assessment of insulin resistance (HOMA-IR) and Matsuda and DeFronzo's insulin sensitivity index (ISI).

Results: PCOS women had higher serum zonulin levels $(P=0.022)$. After adjustment for age and BMI, zonulin levels significantly correlated with HOMA-IR and ISI. Furthermore, PCOS women with more severe menstrual disorders had significantly higher zonulin levels and displayed an inverse correlation between zonulin and the number of menstrual cycles per year $(r=-0.398, P<0.001)$.

Conclusions: Serum zonulin, a biomarker for gut permeability, is increased in PCOS women and correlates with insulin resistance and severity of menstrual disorders. It suggests that alterations in gut permeability may play a role in the pathophysiology of PCOS, and serum zonulin might be used as a biomarker for both risk stratification and therapeutic
\end{abstract} outcomes in PCOS women.

\section{Introduction}

Polycystic ovary syndrome (PCOS) is the most common endocrinopathy in women of reproductive age and is associated with several clinical consequences, including an increased risk for infertility, obesity, insulin resistance, and metabolic alterations. Moreover, these subjects have an increased risk of endometrial carcinoma and cardiovascular disease $(1,2)$. Unfortunately, the exact underlying etiology is presently unknown.
(C) 2015 European Society of Endocrinology Printed in Great Britain
Evidence suggests that increased gut permeability may play a role in the pathogenesis of PCOS (3). Obesity is the most common co-morbidity in PCOS. Both animal models $(4,5)$ and human studies $(6)$ have established that obesity is characterized by increased gut permeability. In addition, irritable bowel syndrome and chronic fatigue syndrome, two conditions linked with increased gut permeability $(7,8)$, are more common in PCOS populations $(9,10)$.

Published by Bioscientifica Ltd 
Furthermore, insulin resistance affects $\sim 70 \%$ of women with PCOS (11), and is associated with increased gut permeability (12). Taken collectively, it is probable that increased gut permeability may contribute to the development of PCOS.

Human zonulin, the eukaryotic counterpart of Vibrio cholerae zonula occludens toxin, is the only physiological mediator currently known to reversibly regulate gut permeability by disassembling intestinal tight junctions $(13,14)$. It increases intestinal permeability in the small intestines and participates in intestinal innate immunity. Zonulin serum concentration is strongly correlated with lactulose: mannitol urine ratio, a widely used clinical indicator of gut permeability (15), and is considered as a non-invasive biomarker for gut permeability $(14,16)$. Previous studies have revealed a key role of zonulindependent gut barrier dysfunction in the pathogenesis of autoimmune diseases (e.g. type 1 diabetes, celiac disease) $(15,17)$. However, the association between zonulin and metabolic diseases has also been recently recognized. Clinical studies have demonstrated that zonulin levels are significantly increased in obese subjects and type 2 diabetes patients, and positively correlates with insulin resistance and inflammatory markers (e.g. tumor necrosis factor $\alpha$, interleukin 6$)(18,19)$. However, the association between zonulin and patients with PCOS remains unknown. This study aimed to determine serum zonulin levels in PCOS and to investigate the potential links between zonulin levels and clinical and biochemical measures of glucose, lipid metabolism, insulin resistance, and severity of menstrual disorders in PCOS subjects.

\section{Subjects and methods}

Seventy-eight newly diagnosed PCOS women were recruited from the endocrine outpatient clinic in Xiangya Hospital of Central South University. The diagnosis of PCOS was established according to the 2003 Rotterdam Consensus Criteria, which require the presence of at least two of the following: oligoovulation and/or anovulation, biochemical and/or clinical signs of hyperandrogenism, polycystic ovaries (by gynecologic ultrasound), after exclusion of other known causes of hyperandrogenemia and ovulatory dysfunction, such as intense physical activity, hypothyroidism, hyperprolactinemia, Cushing's syndrome, congenital adrenal hyperplasia, androgensecreting tumors (20), and those with other concurrent medical illness (kidney, liver disease) also were excluded from the study. PCOS was newly diagnosed in patients who had not previously received any treatment.
The control group were recruited from amongst our hospital staff, and from female patients who were overweight/obese or normal weight with a family history of diabetes, attending our clinic. All of them underwent a $75 \mathrm{~g}$ oral glucose tolerance test (OGTT), and had been tested for luteinizing hormone (LH), follicle-stimulating hormone (FSH), estradiol, prolactin, and total testosterone. It consisted of 63 age-matched healthy women with regular menses $(28 \pm 2$ days) and normal ovaries as assessed by ultrasonography. The participants were excluded if they had thyroid, hepatic, or renal diseases, or took medication known to affect serum sex steroids and blood glucose level for at least 6 months before the start of the study.

A written informed consent for this study was obtained from all the participants, and was approved by the medical Ethics Committee of Central South University Xiangya Hospital.

\section{Anthropometry and laboratory measurements}

Anthropometric data were collected at the time of recruitment. BMI $\left(\mathrm{kg} / \mathrm{m}^{2}\right)$ was calculated based on the height and body weight. According to the Asia-Pacific perspective (21), the degree of obesity was classified as nonobese $\left(B M I<25.0 \mathrm{~kg} / \mathrm{m}^{2}\right)$ or obese $\left(\mathrm{BMI} \geq 25.0 \mathrm{~kg} / \mathrm{m}^{2}\right)$. Waist circumferences (WC) and hip circumferences were measured, starting at the midpoint of the inferior border of the lowest rib and following the iliac crest on the mid-axillary line by around the abdomen, and the waist to hip ratio (WHR) was calculated.

At the time of recruitment, a questionnaire was used to determine the family history and history of diabetes, age of menarche, menstrual cycles per year, and use of oral contraceptives.

A 75-g OGTT was conducted after an 8-12 h overnight fast. The venous blood samples were obtained at 0, 30, 60, and $120 \mathrm{~min}$ after the glucose load to measure glucose and insulin. The serum samples were frozen and stored at $-70{ }^{\circ} \mathrm{C}$ for subsequent zonulin assays.

Plasma glucose, total cholesterol, triglyceride (TG), HDL-C, LDL-C, and uric acid were measured enzymatically on an automatic analyzer (Hitachi) with reagents from Wako Pure Chemical Industries (Osaka, Japan). HbA1c was determined using a high-performance liquid chromatography method on the Bio-Rad D10 Analyzer using reagents from Roche Diagnostics. LH, FSH, estradiol, prolactin, total testosterone, and insulin were measured by commercially available immunochemiluminescent assay (Roche Diagnostic). 
Serum zonulin concentrations were measured in duplicate by using the ELISA Kit (Immundiagnostik AG, Bensheim, Germany). The sensitivity of the assay was $<0.01 \mathrm{ng} / \mathrm{ml}$. Intra- and interassay coefficients of variation for these determinations were between 2.8 and $8.1 \%$ and between 4.8 and $11.6 \%$ respectively. The ELISA Kit used for zonulin measurement only detects the active (uncleaved) form of zonulin.

Visceral adipose index (VAI) was calculated using the proposed formula: $\mathrm{VAI}=\mathrm{WC} /(36.58+(1.89 \times \mathrm{BMI})) \times$ $(\mathrm{TG} / 0.81) \times(1.52 /$ HDL-C) $(22)$. The indexes of homeostasis model assessment of insulin resistance (HOMA-IR) and $\beta$-cell insulin secretion (HOMA- $\beta$ ) were calculated based on the plasma glucose and insulin concentrations as described previously (23). Insulin sensitivity index (ISI) derived from the OGTT was assessed via Matsuda and DeFronzo's ISI (24). $($ ISI $=10000 / \sqrt{ }(($ fasting plasma glucose $\times$ fasting plasma insulin $) \times($ mean glucose $\times$ mean insulin $))$ ).

\section{Statistical analysis}

Statistical analyses were performed using SPSS 19.0 (SPSS). The one-sample Kolmogorov-Smirnov test was used to determine data normality. Normally distributed data were shown as mean \pm s.D., and skewed data were expressed as medians (interquartile range). Differences in clinical characteristics between two groups were analyzed using two-tailed Student's $t$-tests or Mann-Whitney $U$ test where appropriate. Differences among the three groups of patients with PCOS were analyzed using one-way ANOVA or Kruskal-Wallis test Multiple post hoc comparisons were performed using Tukey's correction. Correlation coefficients between serum zonulin with anthropometric parameters and biochemical indexes were determined by simple and partial correlation analyses where appropriate. The Spearman's correlation analysis or partial correlation analysis was applied to assess the correlation of number of menstrual cycles per year along with VAI, HOMA-IR, zonulin and ISI. $P<0.05$ was considered statistically significant.

\section{Results}

\section{Clinical characteristics of study participants}

Clinical characteristics are given in Table 1. Compared with controls, PCOS women had higher systolic blood pressure, WC, WHR, BMI, TGs, VAI, OGTT plasma glucose at $0 \mathrm{~min}\left(\mathrm{Glu}_{0}\right)$ and $120 \mathrm{~min}\left(\mathrm{Glu}_{120}\right)$, OGTT insulin at

Table 1 Clinical and biochemical data of the women with PCOS and control women.

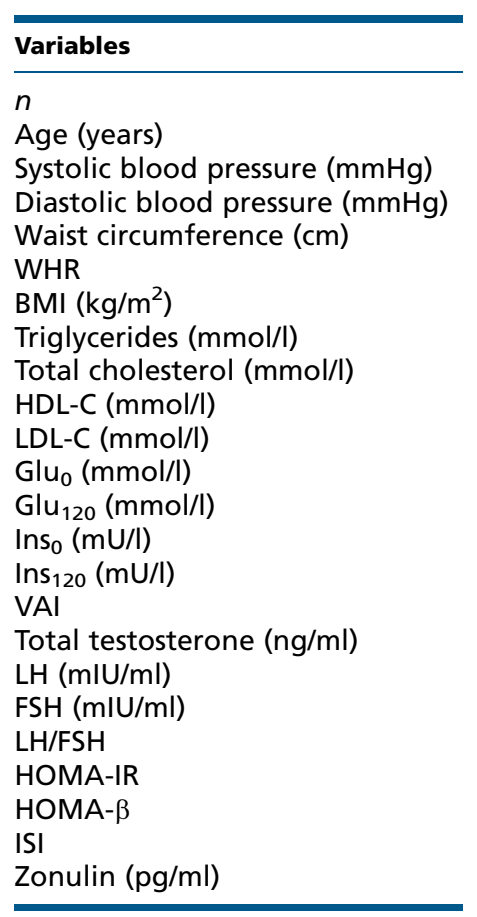

\begin{tabular}{c}
\hline PCOS \\
\hline 78 \\
$29 \pm 5$ \\
$121 \pm 14$ \\
$82 \pm 12$ \\
$91.8 \pm 8.2$ \\
$0.93 \pm 0.05$ \\
$27.6 \pm 4.0$ \\
$2.61 \pm 1.70$ \\
$4.77 \pm 0.86$ \\
$1.33 \pm 0.31$ \\
$2.88 \pm 0.87$ \\
$5.79 \pm 1.82$ \\
$7.63 \pm 3.38$ \\
$15.1(11.2-22.9)$ \\
$70.1(43.1-134.9)$ \\
$3.98 \pm 2.24$ \\
$0.64 \pm 0.29$ \\
$9.05 \pm 5.93$ \\
$5.86 \pm 1.52$ \\
$1.57 \pm 0.94$ \\
$3.91(2.80-7.41)$ \\
$223.8(104.0-250.2)$ \\
$2.53(1.73-3.29)$ \\
$7.78 \pm 5.26$ \\
\end{tabular}

\begin{tabular}{|c|c|}
\hline Controls & $\boldsymbol{P}$ \\
\hline 63 & - \\
\hline $30 \pm 5$ & 0.250 \\
\hline $110 \pm 12$ & 0.021 \\
\hline $77 \pm 7$ & 0.205 \\
\hline $82.3 \pm 8.2$ & 0.003 \\
\hline $0.86 \pm 0.06$ & 0.001 \\
\hline $25.3 \pm 3.0$ & 0.001 \\
\hline $1.44 \pm 1.15$ & 0.023 \\
\hline $4.90 \pm 0.95$ & 0.468 \\
\hline $1.96 \pm 0.38$ & 0.012 \\
\hline $2.42 \pm 0.99$ & 0.065 \\
\hline $5.05 \pm 0.52$ & 0.041 \\
\hline $6.07 \pm 0.99$ & 0.044 \\
\hline $8.0(7.5-11.1)$ & 0.004 \\
\hline $46.6(25.0-60.6)$ & 0.008 \\
\hline $1.61 \pm 1.03$ & 0.015 \\
\hline $0.43 \pm 0.12$ & 0.001 \\
\hline $5.43 \pm 3.12$ & 0.016 \\
\hline $5.92 \pm 1.56$ & 0.651 \\
\hline $0.93 \pm 0.68$ & 0.001 \\
\hline $1.88(1.46-2.51)$ & 0.002 \\
\hline $142.7(93.8-169.6)$ & 0.081 \\
\hline $5.59(2.64-7.87)$ & 0.001 \\
\hline $4.57 \pm 4.05$ & 0.022 \\
\hline
\end{tabular}

Statistically significant $P$ values are in bold. WHR, waist-to-hip ratio; Glu, OGTT plasma glucose 0 min; Glu 120 , OGTT plasma glucose at 120 min; Ins, OGTT insulin at 0 min; Ins $s_{120}$, OGTT insulin at 120 min; VAl, visceral adiposity index; $\mathrm{LH}$, luteinizing hormone; FSH, follicle stimulating hormone; HOMA-IR, HOMA of insulin resistance index; HOMA- $\beta$, HOMA of $\beta$-cell secretion index; ISI, insulin sensitivity index derived from OGTT. 
Table 2 Comparison of clinical parameters between women with PCOS and BMI-matched controls.

\begin{tabular}{|c|c|c|}
\hline & \multicolumn{2}{|c|}{$\operatorname{PCOS}(n=78)$} \\
\hline & Non-obese & Obese \\
\hline$n$ & 21 & 57 \\
\hline Age & $28 \pm 6$ & $29 \pm 5$ \\
\hline $\mathrm{BMI}\left(\mathrm{kg} / \mathrm{m}^{2}\right)$ & $22.5 \pm 2.2$ & $29.3 \pm 3.1$ \\
\hline Waist circumstance (cm) & $81.5 \pm 4.8$ & $95.0 \pm 6.1$ \\
\hline WHR & $0.90 \pm 0.05$ & $0.94 \pm 0.05$ \\
\hline VAI & $2.08 \pm 1.14$ & $4.81 \pm 3.25$ \\
\hline Total testosterone $(\mathrm{ng} / \mathrm{ml})$ & $0.32 \pm 0.12$ & $0.78 \pm 0.45$ \\
\hline HOMA-IR & $2.80(1.40-3.88)$ & $4.60(3.0-7.6)$ \\
\hline HOMA- $\beta$ & $128.3(92-224.4)$ & 246.5 (113.8-366.7) \\
\hline ISI & $3.30(2.21-6.02)$ & $2.42(1.44-2.88)$ \\
\hline Menstrual cycles/year & $10(9-12)$ & $7(4-10)$ \\
\hline Zonulin (pg/ml) & $4.86 \pm 2.26$ & $8.93 \pm 5.56$ \\
\hline
\end{tabular}

\begin{tabular}{c} 
Controls \\
\hline Non-obese \\
\hline 39 \\
$29 \pm 8$ \\
$22.9 \pm 1.7$ \\
$77.6 \pm 5.8$ \\
$0.83 \pm 0.06$ \\
$1.32 \pm 1.02$ \\
$0.31 \pm 0.10$ \\
$1.80(1.19-2.51)$ \\
$123.8(73.4-146.3)$ \\
$5.62(4.64-6.87)$ \\
12 \\
$3.23 \pm 2.18$ \\
\hline
\end{tabular}

s $(n=63)$

Statistically significant $P$ values are in bold. WHR, waist-to-hip ratio; VAl, visceral adiposity index; HOMA-IR, HOMA of insulin resistance index; HOMA- $\beta$, HOMA of $\beta$-cell secretion index; ISI, insulin sensitivity index derived from OGTT; $P 1$, non-obese women with PCOS vs non-obese controls; $P 2$, obese women with PCOS vs obese controls.

0 min $\left(\mathrm{Ins}_{0}\right)$ and $120 \mathrm{~min}\left(\mathrm{Ins}_{120}\right)$, total testosterone, LH and LH/FSH, and a lower HDL-C level $(P<0.05$ for all $)$.

\section{Serum zonulin levels and insulin resistance in PCOS patients and controls}

Serum zonulin levels in PCOS women were significantly higher than those in controls $(P=0.022)$. Compared with controls, the PCOS group also had increased HOMA-IR and decreased ISI, suggesting insulin resistance in PCOS women (Table 1).

After adjusting for BMI, serum zonulin levels, HOMAIR, and ISI in PCOS subjects were still statistically different from those in BMI-matched controls (Table 2).

\section{Relationships between serum zonulin and metabolic parameters}

As given in Table 3, serum zonulin levels correlated significantly with BMI, WHR, TGs, HDL-C, VAI, Glu, $\mathrm{Glu}_{120}$, Ins 0 , Ins ${ }_{120}$, HOMA-IR, and ISI $(P<0.05$ for all). After adjusting for age and BMI, the correlation between serum zonulin levels and HOMA-IR $(r=0.315, P=0.044)$ and ISI $(r=-0.262, P=0.019)$ remained significant.

\section{Relationship between serum zonulin and severity of anovulation}

PCOS patients were classified into three groups according to the severity of menstrual disorders: Group A $(n=23)$ with severe menstrual disorders ( $\leq 6$ cycles/year), Group B $(n=42)$ with mild menstrual disorders (7-10 cycles/year), and Group C $(n=13)$ without menstrual disorders (11-12 cycles/year) (Table 4). Group A subjects had higher blood pressure, WC, BMI, TGs, VAI, glucose levels, insulin, HOMA-IR, and lower HDL-C and ISI, than either groups B

Table 3 Correlation of serum zonulin with anthropometric parameters and biochemical indexes.

\begin{tabular}{|c|c|c|c|c|}
\hline \multirow[b]{2}{*}{ Variables } & \multicolumn{2}{|c|}{ Zonulin } & \multicolumn{2}{|c|}{$\begin{array}{l}\text { Zonulin (adjust for } \\
\text { age and } \mathrm{BMI} \text { ) }\end{array}$} \\
\hline & $R$ & $P$ & $R$ & $P$ \\
\hline Age & -0.019 & 0.702 & - & - \\
\hline BMI & 0.535 & 0.000 & - & - \\
\hline Systolic blood pressure & 0.259 & 0.133 & 0.289 & 0.103 \\
\hline Diastolic blood pressure & 0.260 & 0.131 & 0.279 & 0.116 \\
\hline WHR & 0.401 & 0.015 & -0.015 & 0.935 \\
\hline Triglycerides & 0.422 & 0.031 & -0.091 & 0.673 \\
\hline Total cholesterol & 0.038 & 0.858 & -0.248 & 0.254 \\
\hline HDL-C & -0.412 & 0.031 & -0.239 & 0.272 \\
\hline LDL-C & 0.136 & 0.512 & -0.196 & 0.371 \\
\hline VAI & 0.432 & 0.011 & -0.092 & 0.678 \\
\hline $\mathrm{Glu}_{0}$ & 0.335 & 0.049 & 0.351 & 0.045 \\
\hline $\mathrm{Glu}_{120}$ & 0.445 & 0.007 & 0.347 & 0.045 \\
\hline $\mathrm{Ins}_{0}$ & 0.605 & 0.000 & 0.258 & 0.141 \\
\hline $\operatorname{lns}_{120}$ & 0.527 & 0.001 & 0.246 & 0.161 \\
\hline Total testosterone & 0.411 & 0.051 & 0.01 & 0.972 \\
\hline LH & -0.313 & 0.106 & -0.174 & 0.519 \\
\hline FSH & 0.093 & 0.714 & -0.034 & 0.901 \\
\hline LH/FSH & -0.291 & 0.241 & -0.181 & 0.503 \\
\hline HOMA-IR & 0.645 & 0.000 & 0.315 & 0.044 \\
\hline НОМА- $\beta$ & -0.082 & 0.349 & -0.074 & 0.681 \\
\hline ISI & -0.542 & 0.000 & -0.262 & 0.019 \\
\hline
\end{tabular}

Statistically significant $P$ values are in bold. BMI; WHR, waist-to-hip ratio HDL-C; LDL-C; VAl, visceral adiposity index; Glu 0 , OGTT plasma glucose at $0 \mathrm{~min}$; $\mathrm{Glu}_{120}$, OGTT plasma glucose at $120 \mathrm{~min}$; Ins 0 , OGTT insulin at $0 \mathrm{~min}$; Ins 120 , OGTT insulin at $120 \mathrm{~min}$; $\mathrm{LH}$, luteinizing hormone; $\mathrm{FSH}_{4}$ follicle-stimulating hormone; HOMA-IR, HOMA of insulin resistance index HOMA- $\beta$, HOMA of $\beta$-cell secretion index; ISI, insulin sensitivity index derived from OGTT. 
Table 4 Characteristics of PCOS patients in groups classified by the severity of menstrual disorders.

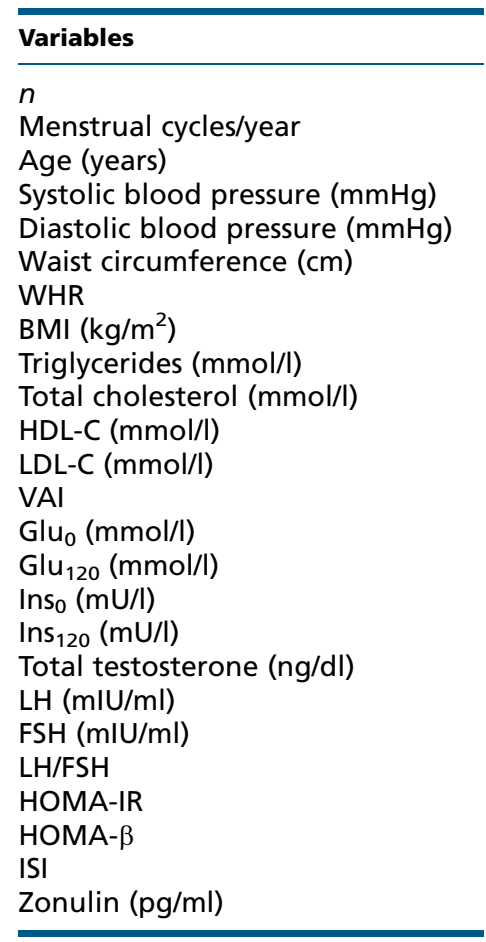

\begin{tabular}{c}
\hline Group A \\
\hline 23 \\
$4(3-6)^{\dagger \S}$ \\
$30 \pm 6$ \\
$133 \pm 15^{* \ddagger}$ \\
$91 \pm 10^{\star \ddagger}$ \\
$95.5 \pm 9.3^{*^{\ddagger}}$ \\
$0.93 \pm 0.05$ \\
$30.4 \pm 4.0^{* \ddagger}$ \\
$3.56 \pm 2.17^{* \ddagger}$ \\
$5.09 \pm 0.93$ \\
$1.15 \pm 0.33^{* \ddagger}$ \\
$3.10 \pm 0.76$ \\
$5.09 \pm 2.04^{\dagger \ddagger}$ \\
$7.30 \pm 2.87^{* \ddagger}$ \\
$8.49 \pm 3.51^{*}$ \\
$29.3(14.1-35.2)^{* \ddagger}$ \\
$149.2(40.5-223.8)^{* \ddagger}$ \\
$0.60 \pm 0.19$ \\
$8.63 \pm 4.41$ \\
$5.69 \pm 1.02$ \\
$1.50 \pm 0.96$ \\
$7.54(4.16-8.29)^{\dagger \ddagger}$ \\
$272.2(47.9-405.7)$ \\
$2.29(1.49-3.46)^{\dagger}$ \\
$10.39 \pm 6.01^{\dagger \ddagger}$ \\
\hline
\end{tabular}

\begin{tabular}{c}
\hline Group B \\
\hline 42 \\
$9(8-10)^{\dagger}$ \\
$28 \pm 5$ \\
$117 \pm 12$ \\
$80 \pm 12$ \\
$90.4 \pm 7.9$ \\
$0.94 \pm 0.04$ \\
$26.6 \pm 3.5$ \\
$2.28 \pm 1.55$ \\
$4.81 \pm 1.01$ \\
$1.38 \pm 0.34$ \\
$2.83 \pm 0.92$ \\
$3.75 \pm 1.79$ \\
$5.22 \pm 0.61$ \\
$7.61 \pm 2.03$ \\
$14.2(10.6-18.9)$ \\
$78.3(46.6-109.9)^{*}$ \\
$0.68 \pm 0.32$ \\
$9.16 \pm 3.34$ \\
$5.88 \pm 1.65$ \\
$1.58 \pm 0.66$ \\
$3.55(2.44-4.69)$ \\
$219.5(100.7-259.8)$ \\
$3.25(2.26-4.80)^{*}$ \\
$7.33 \pm 4.27^{*}$ \\
\hline
\end{tabular}

\begin{tabular}{c}
\hline Group C \\
\hline 13 \\
$11(11-12)$ \\
$30 \pm 4$ \\
$119 \pm 12$ \\
$79 \pm 13$ \\
$90.1 \pm 7.8$ \\
$0.92 \pm 0.05$ \\
$26.4 \pm 4.0$ \\
$2.16 \pm 1.50$ \\
$4.15 \pm 1.18$ \\
$1.43 \pm 0.24$ \\
$2.58 \pm 0.60$ \\
$2.99 \pm 1.96$ \\
$5.07 \pm 0.34$ \\
$6.22 \pm 1.04$ \\
$15.4(6.9-28.8)$ \\
$57.0(13.6-90.4)$ \\
$0.56 \pm 0.29$ \\
$9.44 \pm 4.78$ \\
$6.07 \pm 1.74$ \\
$1.57 \pm 0.65$ \\
$2.77(1.54-3.87)$ \\
$192.8(91.6-421.4)$ \\
$4.43(1.36-7.22)$ \\
$4.99 \pm 2.56$ \\
\hline
\end{tabular}

Compared with group $C,{ }^{*} P<0.05,{ }^{\dagger} P<0.01$; compared with group $B,{ }^{\ddagger} P<0.05,{ }^{\S} P<0.01$; WHR, waist-to-hip ratio; BMI; HDL-C; LDL-C; VAl, visceral adiposity index; Glu

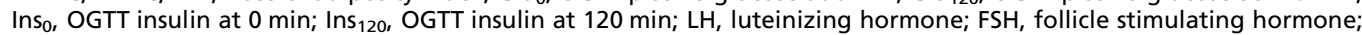
HOMA-IR, HOMA of insulin resistance index; HOMA- $\beta$, HOMA of $\beta$-cell secretion index; ISI, insulin sensitivity index derived from OGTT.

or $\mathrm{C}$, suggesting more severe metabolic dysfunction and insulin resistance in women with more severe menstrual disorders. Of particular interest, serum zonulin level was statistically different between each group (Table 4). In addition, an inverse correlation between zonulin and the number of menstrual cycles per year was observed $(r=-0.398, P<0.001$; Fig. 1$)$. After adjustment for BMI, this correlation remained significant $(r=-0.401, P<0.001)$. However, this inverse correlation was no longer significant after adjustment for HOMA-IR $(r=-0.128, P=0.135)$.

\section{Discussion}

To date, there have been no investigations on the role of zonulin in PCOS. In this study, we showed that serum zonulin levels were significantly elevated in women with PCOS. In addition, we demonstrated a strong relationship between serum zonulin level and obesity, insulin resistance, dyslipidemia, and severity of menstrual disorders.

To our knowledge, this is the first study to illustrate that gut permeability, determined by serum zonulin, is significantly increased in PCOS women. This might be explained by higher prevalence of obesity in the PCOS group, as obesity is characterized by an increased gut permeability $(4,5,6)$, and zonulin levels have been reported to be higher in obese subjects (19). Nevertheless, this study showed a difference in zonulin levels between PCOS women and controls, even after controlling for BMI. Our results suggest that serum zonulin levels are not independently determined by the degree of adiposity in PCOS women. Rather, other contributory factors are likely to play a role. One such factor might be insulin resistance, which was observed in both lean and obese women with PCOS (25), and was confirmed in our study. Our data show PCOS subjects had higher HOMA-IR and lower ISI as compared with BMI-matched controls. In addition, in accordance with Moreno-Navarrete et al. (19), an association between zonulin and insulin resistance was shown in our subjects. Therefore, we speculate that insulin resistance might be a link between elevated zonulin level (increased gut permeability) and development of PCOS. Alterations in gut permeability can compromise the intestinal barrier, allowing access of infectious agents and dietary antigens to facilitate mucosal immune elements, leading to production of inflammatory cytokines. It is well known that inflammatory cytokines, such as interleukin 6 

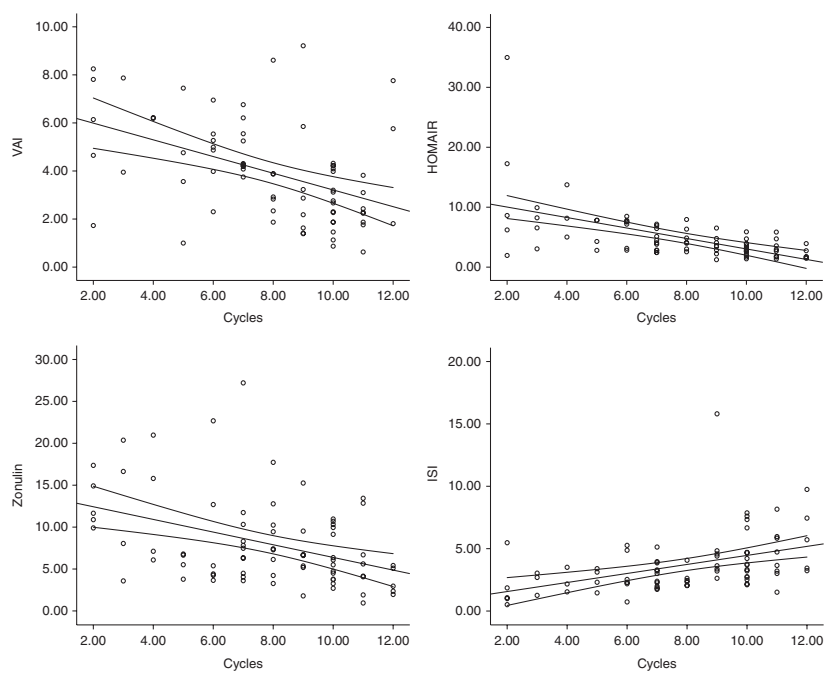

\section{Figure 1}

Correlation of number of menstrual cycles per year with VAI, HOMA-IR, zonulin, and ISI in PCOS women studied. (VAI, visceral adiposity index; HOMA-IR, HOMA of insulin resistance index; ISI, insulin sensitivity index derived from OGTT. With VAI, $r=-0.478, P<0.001$; with HOMA-IR, $r=-0.605, P<0.001$; with ISI, $r=0.552, P<0.001$; with zonulin, $r=-0.398, P<0.001)$.

(IL6) and $\mathrm{TNF} \alpha$, can lead to insulin resistance (26). In addition, cytokines control zonulin production, as the HP2 gene (which encodes zonulin production) promoter is under IL6 control through STAT3 activation and miR-18a induction (27). Thus, increased gut permeability leads to insulin resistance through a continuous inflammatory state.

In this study, we found PCOS women with more severe menstrual disorders had significantly higher zonulin levels, as well as an inverse correlation between zonulin and the number of menstrual cycles per year indicates an association between zonulin levels and severity of menstrual disorders. However, the correlation between serum zonulin and the number of menstrual cycles per year was no longer significant after adjusting for HOMA-IR. This suggests that insulin resistance is an essential link between zonulin and severity of menstrual disorders. In support of this, our data also show an inverse correlation between the number of menstrual cycles per year and insulin resistance, as estimated by HOMA-IR and ISI (Fig. 1).

Visceral adiposity index (VAI), a surrogate marker of adipose tissue function and distribution, is strongly associated with visceral adipose (22). VAI could be a useful tool for the assessment of cardiometabolic risk associated with PCOS (28) in both clinical settings and in population studies. This study showed a strong relationship between serum zonulin level and VAI, suggesting serum zonulin may be valuable to detect patients at high risk to develop cardiometabolic disease.

Several animal studies have shown that prebiotic and probiotic supplements can help to improve intestinal barrier function and prevent impaired insulin resistance $(29,30)$. Therefore, we may expect that prebiotic and probiotic supplements would be an effective treatment for PCOS.

Hyperandrogenemia is the biochemical hallmark of PCOS (31). It was proposed that hyperandrogenemism can induce insulin resistance in PCOS, which in turn also plays a role in the development of hyperandrogenemism $(32,33)$. Hyperandrogenemia and ovulatory disturbances are commonly encountered in the syndromes of extreme insulin resistance (34). We failed to identify any association between total testosterone and zonulin level in our study. This may be a result of poor sensitivity of the methods used for total testosterone assays. The free androgen index may be more precise for hyperandrogenemia $(35,36)$.

Our study is not without limitations. The total number of participants was limited. Also, it would be appropriate to match groups for age and BMI (and not just for age), although we tried to overcome this limitation by using multivariate correction in the analysis of the correlation between zonulin with anthropometric and biochemical parameters. Also, given the cross-sectional nature, our study does not prove a mechanistic link between zonulin (increased gut permeability) and the development of PCOS. In addition, as increased gut permeability leads to insulin resistance through a continuous inflammatory state, it would be noteworthy to measure related cytokines, such as interleukin $1 \beta$, tumor necrosis factor $\alpha$, interleukin 6 , in order to further explore the potential mechanisms between zonulin and PCOS.

In conclusion, our results show that serum levels of zonulin, a biomarker for gut permeability, were increased in PCOS women, and correlated with insulin resistance and severity of menstrual disorders. It suggested that alterations in gut permeability may play a role in the pathophysiology of PCOS, and serum zonulin might be used as a marker for both risk stratification and therapeutic outcomes in PCOS women.

Declaration of interest

The authors declare that there is no conflict of interest that could be perceived as prejudicing the impartiality of the research reported. 


\section{Funding}

This research did not receive any specific grant from any funding agency in the public, commercial or not-for-profit sector.

\section{Author contribution statement}

All authors listed in this manuscript contributed significantly to the development of the research and writing of the manuscript. Dr D Zhang designed the study. Drs L Zhang, F Yue, and Y Zheng contributed to sample collecting, data collection, and testing. Dr R Russell contributed to data analyses. Drs $D$ Zhang and $R$ Russell prepared the manuscript for submission.

\section{Acknowledgements}

The authors are indebted to all subjects for agreeing to participate in this study.

\section{References}

1 Azziz R, Carmina E, Dewailly D, Diamanti-Kandarakis E, EscobarMorreale HF, Futterweit W, Janssen OE, Legro RS, Norman RJ, Taylor AE et al. The Androgen Excess and PCOS Society criteria for the polycystic ovary syndrome: the complete task force report. Fertility and Sterility 200991 456-488. (doi:10.1016/j.fertnstert.2008.06.035)

2 Vrbikova J, Grimmichova T, Dvorakova K, Hill M, Stanicka S \& Vondra K. Family history of diabetes mellitus determines insulin sensitivity and $\beta$ cell function in polycystic ovary syndrome. Physiological Research 200857 547-553. (doi: 10.1080/ 09513590902972133)

3 Tremellen K \& Pearce K. Dysbiosis of gut microbiota (DOGMA) - a novel theory for the development of polycystic ovarian syndrome. Medical Hypotheses 201279 104-112. (doi:10.1016/j.mehy.2012.04.016)

4 Cani PD, Bibiloni R, Knauf C, Waget A, Neyrinck AM, Delzenne NM \& Burcelin R. Changes in gut microbiota control metabolic endotoxemiainduced inflammation in high-fat diet-induced obesity and diabetes in mice. Diabetes 200857 1470-1481. (doi:10.2337/db07-1403)

5 Cani PD, Possemiers S, Van de Wiele T, Guiot Y, Everard A, Rottier O, Geurts L, Naslain D, Neyrinck A, Lambert DM et al. Changes in gut microbiota control inflammation in obese mice through a mechanism involving GLP-2-driven improvement of gut permeability. Gut $2009 \mathbf{5 8}$ 1091-1103. (doi:10.1136/gut.2008.165886)

6 Gummesson A, Carlsson LM, Storlien LH, Backhed F, Lundin P, Lofgren L, Stenlof K, Lam YY, Fagerberg B \& Carlsson B. Intestinal permeability is associated with visceral adiposity in healthy women. Obesity 201119 2280-2282. (doi:10.1038/oby.2011.251)

7 Piche T, Barbara G, Aubert P, Bruley des Varannes S, Dainese R, Nano JL, Cremon C, Stanghellini V, De Giorgio R, Galmiche JP et al. Impaired intestinal barrier integrity in the colon of patients with irritable bowel syndrome: involvement of soluble mediators. Gut 200958 196-201. (doi:10.1136/gut.2007.140806)

8 Maes M, Twisk FN, Kubera M \& Ringel K. Evidence for inflammation and activation of cell-mediated immunity in myalgic encephalomyelitis/chronic fatigue syndrome (ME/CFS): increased interleukin-1, tumor necrosis factor- $\alpha$, PMN-elastase, lysozyme and neopterin. Journal of Affective Disorders 2012136 933-939. (doi:10.1016/j.jad.2011. 09.004)

9 Mathur R, Ko A, Hwang LJ, Low K, Azziz R \& Pimentel M. Polycystic ovary syndrome is associated with an increased prevalence of irritable bowel syndrome. Digestive Diseases and Sciences 201055 1085-1089. (doi:10.1007/s10620-009-0890-5)
10 Harlow BL, Signorello LB, Hall JE, Dailey C \& Komaroff AL. Reproductive correlates of chronic fatigue syndrome. American Journal of Medicine 1998105 94S-99S. (doi:10.1016/S0002-9343(98)00173-9)

11 Moghetti P, Tosi F, Bonin C, Di Sarra D, Fiers T, Kaufman JM, Giagulli VA, Signori C, Zambotti F, Dall'Alda M et al. Divergences in insulin resistance between the different phenotypes of the polycystic ovary syndrome. Journal of Clinical Endocrinology and Metabolism 2013 98 E628-E637. (doi:10.1210/jc.2012-3908)

12 de Kort S, Keszthelyi D \& Masclee AA. Leaky gut and diabetes mellitus: what is the link? Obesity Reviews 201112 449-458. (doi:10.1111/j.1467789X.2010.00845.x)

13 Fasano A. Zonulin, regulation of tight junctions, and autoimmune diseases. Annals of the New York Academy of Sciences 20121258 25-33. (doi:10.1111/j.1749-6632.2012.06538.x)

14 Wang W, Uzzau S, Goldblum SE \& Fasano A. Human zonulin, a potential modulator of intestinal tight junctions. Journal of Cell Science $20001134435-4440$.

15 Sapone A, de Magistris L, Pietzak M, Clemente MG, Tripathi A, Cucca F, Lampis R, Kryszak D, Carteni M, Generoso M et al. Zonulin upregulation is associated with increased gut permeability in subjects with type 1 diabetes and their relatives. Diabetes 200655 1443-1449. (doi:10.2337/db05-1593)

16 Smecuol E, Sugai E, Niveloni S, Vazquez H, Pedreira S, Mazure R, Moreno ML, Label M, Maurino E, Fasano A et al. Permeability, zonulin production, and enteropathy in dermatitis herpetiformis. Clinical Gastroenterology and Hepatology 20053 335-341. (doi:10.1016/S15423565(04)00778-5)

17 Fasano A. Celiac disease - how to handle a clinical chameleon. New England Journal of Medicine 2003348 2568-2570. (doi:10.1056/ NEJMe030050)

18 Jayashree B, Bibin YS, Prabhu D, Shanthirani CS, Gokulakrishnan K, Lakshmi BS, Mohan V \& Balasubramanyam M. Increased circulatory levels of lipopolysaccharide (LPS) and zonulin signify novel biomarkers of proinflammation in patients with type 2 diabetes. Molecular and Cellular Biochemistry 2014388 203-210. (doi:10.1007/s11010-0131911-4)

19 Moreno-Navarrete JM, Sabater M, Ortega F, Ricart W \& FernandezReal JM. Circulating zonulin, a marker of intestinal permeability, is increased in association with obesity-associated insulin resistance. PLOS ONE 20127 e37160. (doi:10.1371/journal.pone.0037160)

20 Rotterdam ESHRE/ASRM-Sponsored PCOS Consensus Workshop Group. Revised 2003 consensus on diagnostic criteria and long-term health risks related to polycystic ovary syndrome. Fertility and Sterility 200481 19-25. (doi:10.1016/j.fertnstert.2003.10.004)

21 WHO Expert Consultation. Appropriate body-mass index for Asian populations and its implications for policy and intervention strategies. Lancet 2004363 157-163. (doi:10.1016/S0140-6736(03)15268-3)

22 Amato MC, Giordano C, Galia M, Criscimanna A, Vitabile S, Midiri M, Galluzzo A \& AlkaMeSy Study Group. Visceral adiposity index: a reliable indicator of visceral fat function associated with cardiometabolic risk. Diabetes Care 201033 920-922. (doi:10.2337/dc09-1825)

23 Matthews DR, Hosker JP, Rudenski AS, Naylor BA, Treacher DF \& Turner RC. Homeostasis model assessment: insulin resistance and $\beta$-cell function from fasting plasma glucose and insulin concentrations in man. Diabetologia 198528 412-419. (doi:10.1007/BF00280883)

24 Matsuda M \& DeFronzo RA. Insulin sensitivity indices obtained from oral glucose tolerance testing: comparison with the euglycemic insulin clamp. Diabetes Care 199922 1462-1470. (doi:10.2337/diacare.22.9. 1462)

25 Goodarzi MO \& Korenman SG. The importance of insulin resistance in polycystic ovary syndrome. Fertility and Sterility $2003 \mathbf{8 0} 255-258$. (doi:10.1016/S0015-0282(03)00734-9)

26 Shoelson SE, Lee J \& Goldfine AB. Inflammation and insulin resistance. Journal of Clinical Investigation 2006116 1793-1801. (doi:10.1172/ JCI29069) 
27 Brock M, Trenkmann M, Gay RE, Gay S, Speich R \& Huber LC. MicroRNA-18a enhances the interleukin-6-mediated production of the acute-phase proteins fibrinogen and haptoglobin in human hepatocytes. Journal of Biological Chemistry $201128640142-40150$. (doi:10.1074/jbc.M111.251793)

28 Amato MC, Verghi M, Galluzzo A \& Giordano C. The oligomenorrhoic phenotypes of polycystic ovary syndrome are characterized by a high visceral adiposity index: a likely condition of cardiometabolic risk. Human Reproduction 201126 1486-1494. (doi:10.1093/humrep/ der088)

29 Ma X, Hua J \& Li Z. Probiotics improve high fat diet-induced hepatic steatosis and insulin resistance by increasing hepatic NKT cells. Journal of Hepatology 200849 821-830. (doi:10.1016/j.jhep.2008. 05.025)

30 Everard A, Lazarevic V, Derrien M, Girard M, Muccioli GG, Neyrinck AM, Possemiers S, Van Holle A, Francois P, de Vos WM et al. Responses of gut microbiota and glucose and lipid metabolism to prebiotics in genetic obese and diet-induced leptin-resistant mice. Diabetes 201160 2775-2786. (doi:10.2337/db11-0227)

31 Wild RA, Carmina E, Diamanti-Kandarakis E, Dokras A, EscobarMorreale HF, Futterweit W, Lobo R, Norman RJ, Talbott E \& Dumesic DA. Assessment of cardiovascular risk and prevention of cardiovascular disease in women with the polycystic ovary syndrome: a consensus statement by the Androgen Excess and Polycystic Ovary Syndrome (AE-PCOS) Society. Journal of Clinical Endocrinology and Metabolism 201095 2038-2049. (doi:10.1210/jc.2009-2724)
32 Burghen GA, Givens JR \& Kitabchi AE. Correlation of hyperandrogenism with hyperinsulinism in polycystic ovarian disease. Journal of Clinical Endocrinology and Metabolism 198050 113-116. (doi:10.1210/ jcem-50-1-113)

33 Diamanti-Kandarakis E \& Dunaif A. Insulin resistance and the polycystic ovary syndrome revisited: an update on mechanisms and implications. Endocrine Reviews 201233 981-1030. (doi:10.1210/ er.2011-1034)

34 Alvarez-Blasco F, Botella-Carretero JI, San Millan JL \& EscobarMorreale HF. Prevalence and characteristics of the polycystic ovary syndrome in overweight and obese women. Archives of Internal Medicine 2006166 2081-2086. (doi:10.1001/archinte.166.19.2081)

35 Bhasin S, Zhang A, Coviello A, Jasuja R, Ulloor J, Singh R, Vesper H \& Vasan RS. The impact of assay quality and reference ranges on clinical decision making in the diagnosis of androgen disorders. Steroids 2008 73 1311-1317. (doi:10.1016/j.steroids.2008.07.003)

36 Rosner W, Vesper H \& Endocrine Society, American Association for Clinical Chemistry, American Association of Clinical Endocrinologists, Androgen Excess/PCOS Society, American Society for Bone and Mineral Research, American Society for Reproductive Medicine, American Urological Association, Association of Public Health Laboratories, Endocrine Society, Laboratory Corporation of America, North American Menopause Society, Pediatric Endocrine Society. Toward excellence in testosterone testing: a consensus statement. Journal of Clinical Endocrinology and Metabolism 201095 4542-4548. (doi:10.1210/jc.2010-1314)

Received 14 July 2014

Revised version received 1 October 2014

Accepted 20 October 2014 